\title{
LOS ASENTAMIENTOS PREHISPÁNICOS Y LA ARQUITECTURA EN LA ISLA CAN CUN, QUINTANA ROO*
}

\author{
Ernesto Vargas Pacheco \\ Centro de Estudios Mayas
}

En este trabajo intento hacer una revisión de las exploraciones e investigaciones arqueológicas de los sitios mayas localizados en la isla Can Cun, Quintana Roo, ubicada a $21^{\circ} 08^{\prime}$ de la latitud norte y $86^{\circ} 46^{\prime}$ de longitud oeste. La isla mide $21 \mathrm{kms}$. de largo por 400 metros en su parte más ancha; el estudio se limita especialmente a esta área y temporalmente a la problemática del desarrollo histórico de la tradición arquitectónica denominada Costa Oriental.

En la primera parte del trabajo expongo los datos aportados por viajeros y exploradores que visitaron la isla de Can Cun. En la segunda describo las estructuras aisladas y los sitios arqueológicos que se encuentran en diversos lugares de la isla. Después, por considerarlo de interés, lo hago en forma particular en los sitios El Rey y San Miguel, señalando las características arquitectónicas más importantes. Por último, planteo algunas relaciones con otros sitios y anoto ciertos problemas de importancia.

Antecedentes históricos. En los trabajos de Lothrop (1924), Edwards (1957), y Andrews IV (1975), aparecen historias y reportes de los visitadores de la Costa Oriental de Quintana Roo. Citaré, sin embargo, solamente las referencias de aquellos que visitaron la isla y/o dejaron información de los vestigios arqueológicos de la misma.

* El Proyecto Can Cun fue dirigido por el arqueólogo Norberto González, y el trabajo de campo estuvo a cargo de Pablo Mayer. Centro Regional del Sureste, INAH. Este ensayo es parte de los resultados obtenidos durante mi participación en el Proyecto, agradezco a Akira Kaneco su colaboración en los planos, y al arqueólogo Lorenzo Ochoa S. del C.E.M. sus atinados comentarios y correcciones. 
Por otra parte, debo subrayar que no existen noticias sobre esta isla en ninguna de las fuentes históricas conocidas, solamente se sabe que se encontraba en la provincia de Ecab; tampoco se sabe si el nombre contemporáneo de Can Cun o Can cuen es el mismo que tenía en tiempos prehispánicos.

$\mathrm{El}$ primer reporte sobre la isla se atribuye al capitán inglés $\mathrm{Ri}$ chard Owen, pero no menciona nada sobre restos de la ocupación prehispánica; sin embargo, hizo observaciones de gran interés al señalar, entre otras cosas, la existencia de pozos de agua dulce en las puntas norte y sur, en los que se abastecían los pescadores.

John Stephen es el primero que reporta asentamientos prehispánicos en la isla de Can Cun; él navegó a lo largo de la costa y pasó la noche en Punta Nisuc. Sin lugar a duda visitó o vio los templos de la punta Can Cun, el templo costero o Yamilum, el templo de San Miguel que está sobre la duna y los de Punta Nisuc. Anotó que los dos templos de la Punta Can Cun se encontraban bastante deteriorados y que se asemejaban mucho a los de Tulum.

Entre los años de 1877 y 1878 Alicia y Augusto Le Plongeon viajan a lo largo de la costa norte de Quintana Roo visitando y describiendo las ruinas arqueológicas de Isla Mujeres, Can Cun, El Meco y Cozumel. La descripción que hacen de las ruinas de Nisuc té de la isla de Can Cun, posiblemente se refiere al sitio conocido hoy como El Rey.

William H. Holmes, en enero de 1895, después de rodear la punta norte de la isla - punta Can Cun- llega hasta los templos visitados por Stephens; después encausa por el canal a la laguna y visita una ranchería que tenía cocales y milpas de maiz; en las cercanías observó un sitio con muchos edificios de piedra y estructuras caídas y señaló que tenían columnas y cuartos; sin embargo, aunque no hace mención del nombre, posiblemente se refiere al sitio conocido hoy como San Miguel.

Los ingleses Arnold y Frost visitaron San Miguel, El Rey y Punta Nisuc en 1909, levantaron el primer plano del sitio conocido como El Rey, con la estructura piramidal, la estructura de la bóveda y dos plataformas con columnas (figura 1). Asimismo describen los restos de una figura antropomorfa, conocida posteriormente con el nombre de El Rey (Arnold y Frost 1909).

En 1924 se editan los libros de Lothrop y Gann, en donde el primero presenta una buena descripción de las ruinas de El Rey, al sintetizar los datos de Arnold y Frost con los de Morley y Gann, que habían visitado la isla en 1918. Reproduce el plano de Arnold 


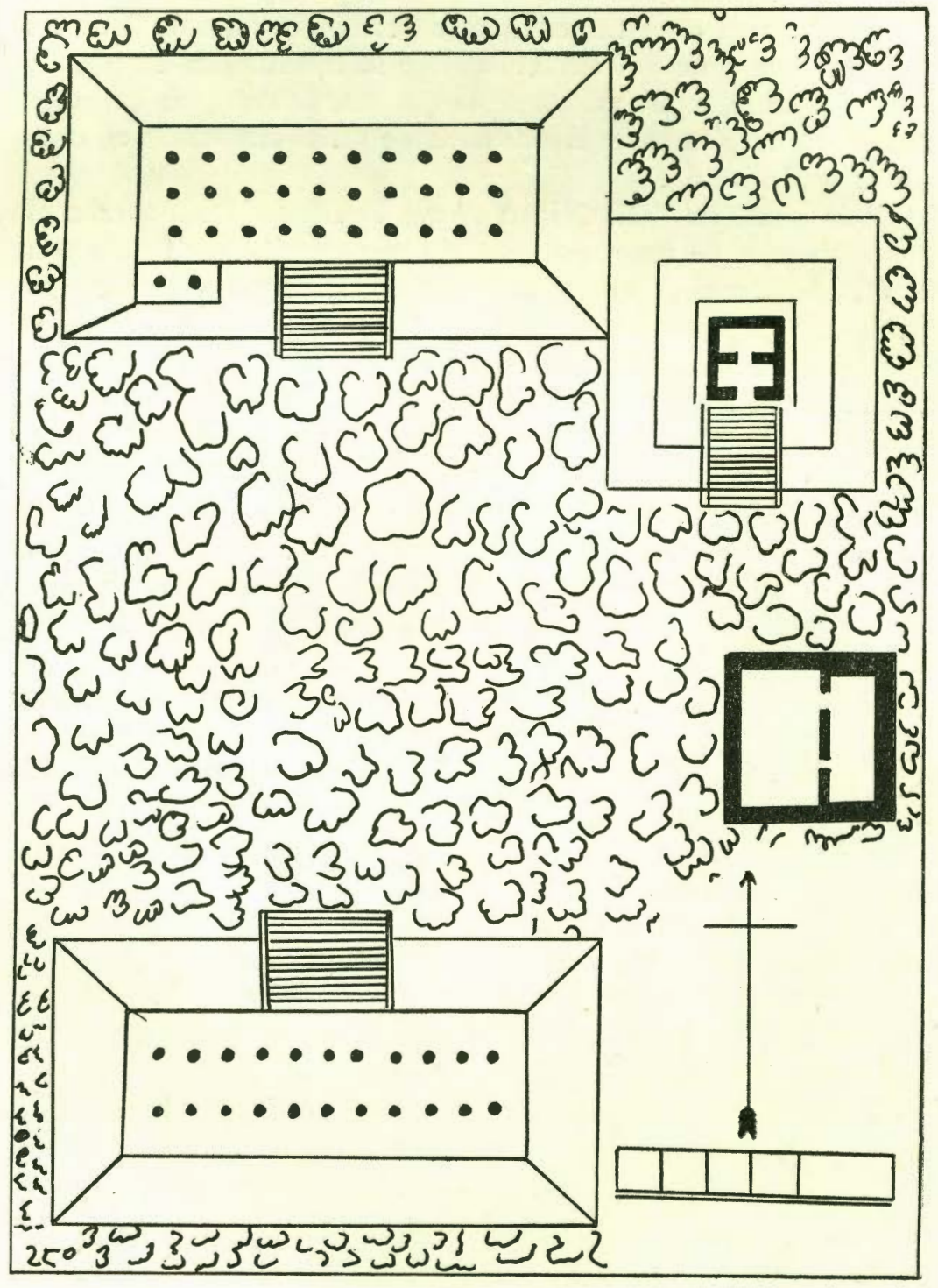

MAPA DE LAS RUINAS DE CAN CUN (TOMADO DE ARNOLDY FROST 1909:151)

F | G. I 
y Frost y agrega otros tres edificios, así como dos fotos de la cabeza antropomorfa y de dos estructuras que se encontraban en pie. El segundo, Gann, repite mucho de lo que dice Lothrop, sin embargo, añade información sobre la existencia de varias estructuras en otras partes de la isla.

Más tarde, en 1938, Miguel Ảngel Fernández llevó a cabo algunos trabajos de reconocimiento y reconstrucción en Tulum y en la Isla de Cozumel, observando que para él existe una diferencia en los templos mayas del antiguo imperio y los de Tulum, ya que en los primeros domina el macizo sobre el claro y nunca se usó columna. De esto dedujo que se pueden establecer dos grupos: uno típicamente maya y otro donde predomina el elemento columna. Cabe la pena destacar lo que nos dice sobre Cancun: "isla situada a $25 \mathrm{kms}$. aproximadamente al sur de isla Mujeres, hay un caso similar con siete columnas al frente y nueve interiores, solo que aquí no existe la galería posterior; la cornisa es también de tres elementos como el de la subestructura de Tulum" (Fernández 1945: 99-100).

Durante 1954 W.T. Sanders trabajó en Quintana Roo e hizo algunos pozos en los sitios de San Miguel y El Rey; no publicó ningún plano, aunque señala lo incompleto del mapa de Arnold y Frost. En su mayoría la cerámica pertenece al periodo Tulum, sin embargo, sugiere la posibilidad de una ocupación anterior (Sanders, W., 1955). Podemos decir, por lo tanto, que la ocupación de El Rey y San Miguel se pueden señalar en el periodo Floreciente modificado y en el periodo Decadente.

En 1963 bajo la dirección de Andrews IV se excava El Conchero en la playa norte de la isla, que resulta ser un campamento de pescadores del formativo tardío. Reporta también dos estructuras en la punta norte, asignándolas al periodo decadente (Andrews IV et al. 1974).

Asentamientos Prehispánicos. En la primera parte de este trabajo he presentado la información disponible de los sitios a que hacen referencia diferentes autores. Existe, como se ha podido constatar, falta de claridad en las referencias; utilizan indistintamente los nombres de Can Cun, Cancuen, Nisucté y Punta Nisuc para los diversos asentamientos prehispánicos localizados en la isla.

A continuación describiré los sitios arqueológicos y las estructuras aisladas que se encuentran distribuidas en la isla: (figura 2)

1. Koxolnah o El Table, es un pequeño sitio del preclásico superior, ubicado en tierra firme y que da hacia la laguna. Está 


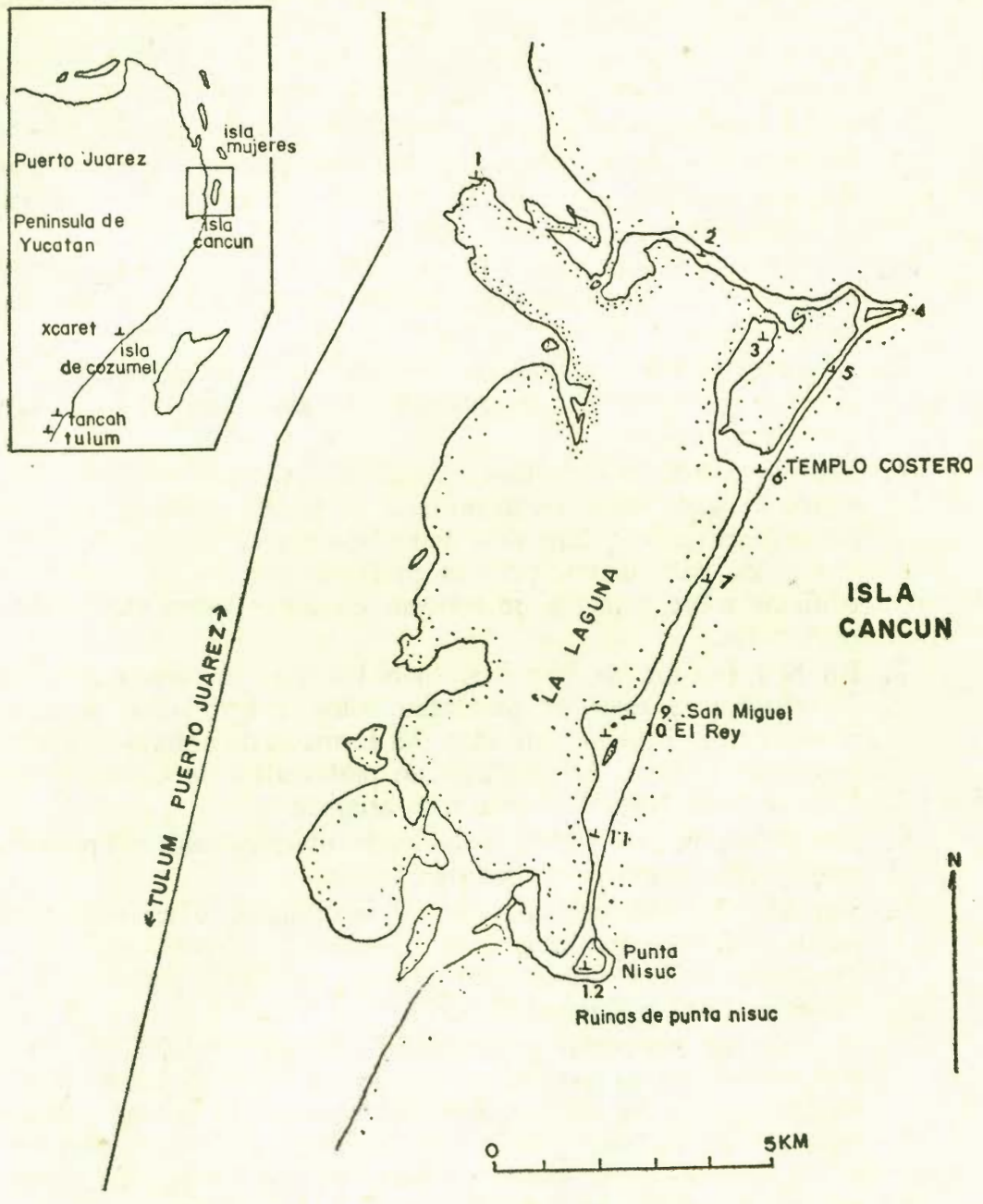

MAPA DE CANCUN, QR, MEXICO (ADAPTADO DE WARD: 1973,FIGI)

LA LOCALIZACION DE SITIOS ES APROXIMADA

(MAPA TOMADO DE ANDREWS II ET, AL., 1974)

F1G.2 
formado por 12 estructuras de tamaño y alturas diferentes, y delimitado por un pequeño muro de piedras; se hizo el levantamiento topográfico del sitio y pozos estratigráficos. Por la cerámica obtenida, se supone cierta contemporaneidad de este sitio con El Conchero excavado por Andrews IV.

2. En la isla la primera evidencia se encuentra en Villas Ticul. Son restos de una plataforma semidestruida por las construcciones modernas; corresponde posiblemente al postclásico tardío.

3. Hacia la laguna existen dos estructuras exploradas y consolidadas que pertenecen al postclásico tardío; se localizan en lo que es actualmente el campo de golf.

4. En el lugar conocido como Punta Can Cun existen dos pequeños templos sobre el acantilado; tienen arquitectura característica del postclásico tardío.

5. El Conchero está ubicado en la playa norte mirando hacia el mar; se trata de un campamento de pescadores del periodo formativo tardío.

6. Templo costero o Yamilum; consiste en dos templos construidos frente al mar, sobre un acantilado de piedra arenisca; son del postclásico tardío y han sido destruidos por el saqueo. No existen datos particulares, pero es probable que los que reportan edificios sobre la costa, se refieran en algún momento a estas estructuras.

7. En San Joaquín se han localizado hasta el momento más de 20 chultunes; estos se presentan solos o agrupados; algunos de ellos están intercomunicados. La forma es de botellón y varios conservan la tapa. Se localizan en partes altas de la isla, hacia el lado de la laguna y sobre roca arenisca.

8. San Pablo, pequeño templo destruido ubicado sobre una duna, corresponde también al a misma época.

9. San Miguel, sitio con más de 30 estructuras. Haré posteriormente una descripción general, adjuntando el plano del sitio. Posiblemente fue visitado por Holmes en 1895, por Arnold y Frost en 1909 y Sanders en 1954.

10. El Rey fue explorado y consolidado en su totalidad; de éste presento el plano general y algunos detalles arquitectónicos. Históricamente ha sido el sitio más visitado de la isla; existen informes en Salisbury, 1877, en Spinden, 1913, Le Plongeon, 1889, Holmes, 1895, Arnold y Frost, 1909, Lothrop, 1924, Sand. ers, 1954 y 1960, Andrews IV et al., 1974 etcétera.

Relacionados con el sitio, sobre un islote de piedra arenisca, 200 metros al oeste existen unos chultunes en forma de botellones intercomunicados de los cuales uno conserva todavía la tapa original.

11. Entre El Rey y Punta Nisuc, hacia la laguna y muy cerca de ella, se encuentran dos pequeños edificios destruidos, característicos de la arquitectura de la costa oriental. Se han localizado restos de altares saqueados, denotándose su existencia tan solo por el amontonamiento de unas cuantas piedras y restos de estuco. 
12. De Punta Nisuc se tienen noticias de la existencia de construcciones prehispánicas en los trabajos de Stephens, Arnold y Frost, Lothrop y Atlas Arqueológico de la República Mexicana; no se han localizado quizás porque fueron destruidos al construirse un hotel.

El Rey y San Miguel. Los pobladores de El Rey y San Miguel escogieron para asentarse una de las partes más anchas de la isla, en donde aprovecharon maravillosamente su topografía; el terreno fue transformado artificialmente por las plataformas para la ubicación de las plazas, estructuras ceremoniales y civiles. Para ello utilizaron la materia prima de los alrededores de la isla y tierra firme.

En ambos sitios se aprecian una serie de elementos arquitectónicos y urbanísticos que indican el sentido de planeación, aprovechamiento del lugar, conocimientos constructivos y tradición estilística, mismos que se reflejan en los espacios abiertos como plazas y caminos. Los conocimientos constructivos quedan de manifiesto en la utilización de la materia prima del lugar en pisos, columnas, techos, rellenos etcétera, y la tradición estilística se aprecia en los detalles arquitectónicos de los edificios y plataformas.

Para el estudio de los asentamientos arqueológicos de El Rey seguiré la división que se hizo durante su exploración y consolidación, aprovechando además los nombres locales de El Rey y Pinturas; el primero, como ya indiqué, se debe al descubrimiento de una cabeza antropomorfa y el segundo, que está al sur, se debe al hallazgo de fragmentos de estuco pintado.

El Rey. Es conocido también con los nombres de Can Cun, Cancuen, Nisucté y Punta Nisuc; indistintamente aplicaban estos nombres a casi todos las ruinas del sur de la isla, y por consiguiente los conocidos como Punta Nisuc, El Rey y San Miguel, fueron previamente reportados bajo esos nombres, dificultándose en la actualidad determinar a qué sitio se referían.

El sitio de El Rey se encuentra a $2.5 \mathrm{kms}$. al norte de Punta Nisuc. Su distribución se ajusta a la forma y topografía de la isla, con una extensión de 520 metros de norte a sur y 70 metros en su parte más ancha; sobre esta superficie se construyeron 47 estructuras.

En los trabajos de exploración y consolidación, el sitio fue dividido en dos conjuntos: El Rey y Pinturas. Las exploraciones se iniciaron en El Rey, que es el conjunto central. Allí se localizan las estructuras principales, 7 en total, más 9 plataformas en la parte norte y 27 en Pinturas (plang 1 ). 
Como indiqué, en la parte central se concentran los principales edificios del sitio: una estructura piramidal, compuesta de tres cuerpos en talud, esquinas redondeadas y un templo en la parte superior, la estructura 3 que es una bóveda escalonada y en su interior conserva pinturas murales muy deterioradas, allí mismo se observan 6 piedras asentadas en el piso a manera de asientos (figura 3). Otro edificio importante de la parte central de El Rey son las grandes plataformas que varían en forma y tamaño, pero se caracterizan por tener doble hilera de columnas que sostenían un techo plano; en el interior existe una banqueta y un altar central (figura 4). En la estructura 4 además de una alfarda con dado,

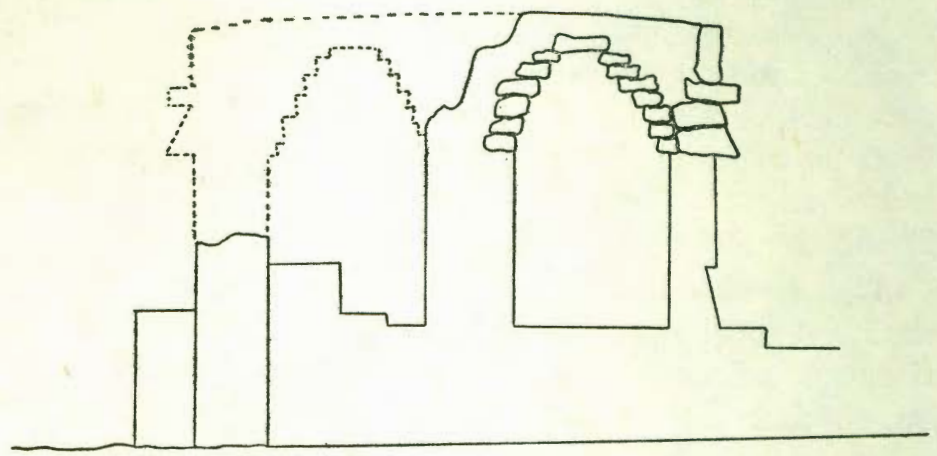

CORTE DE IA BOVEDA

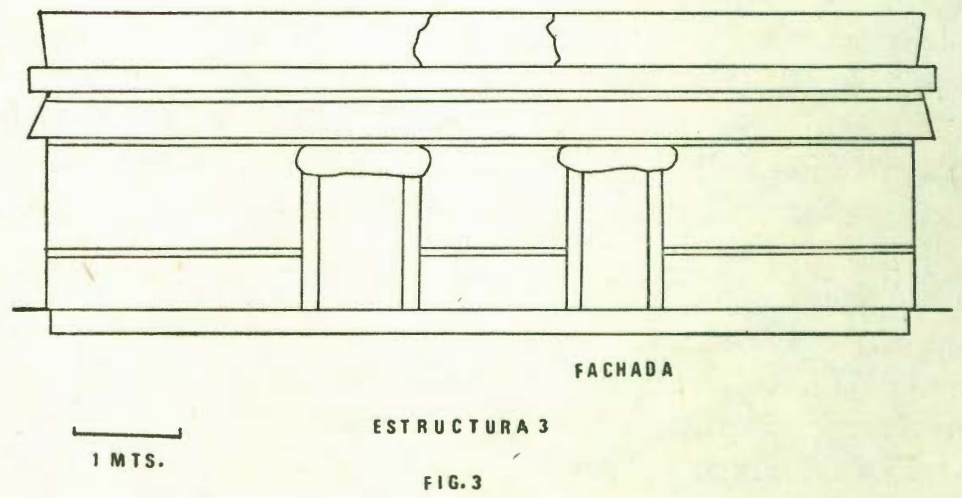



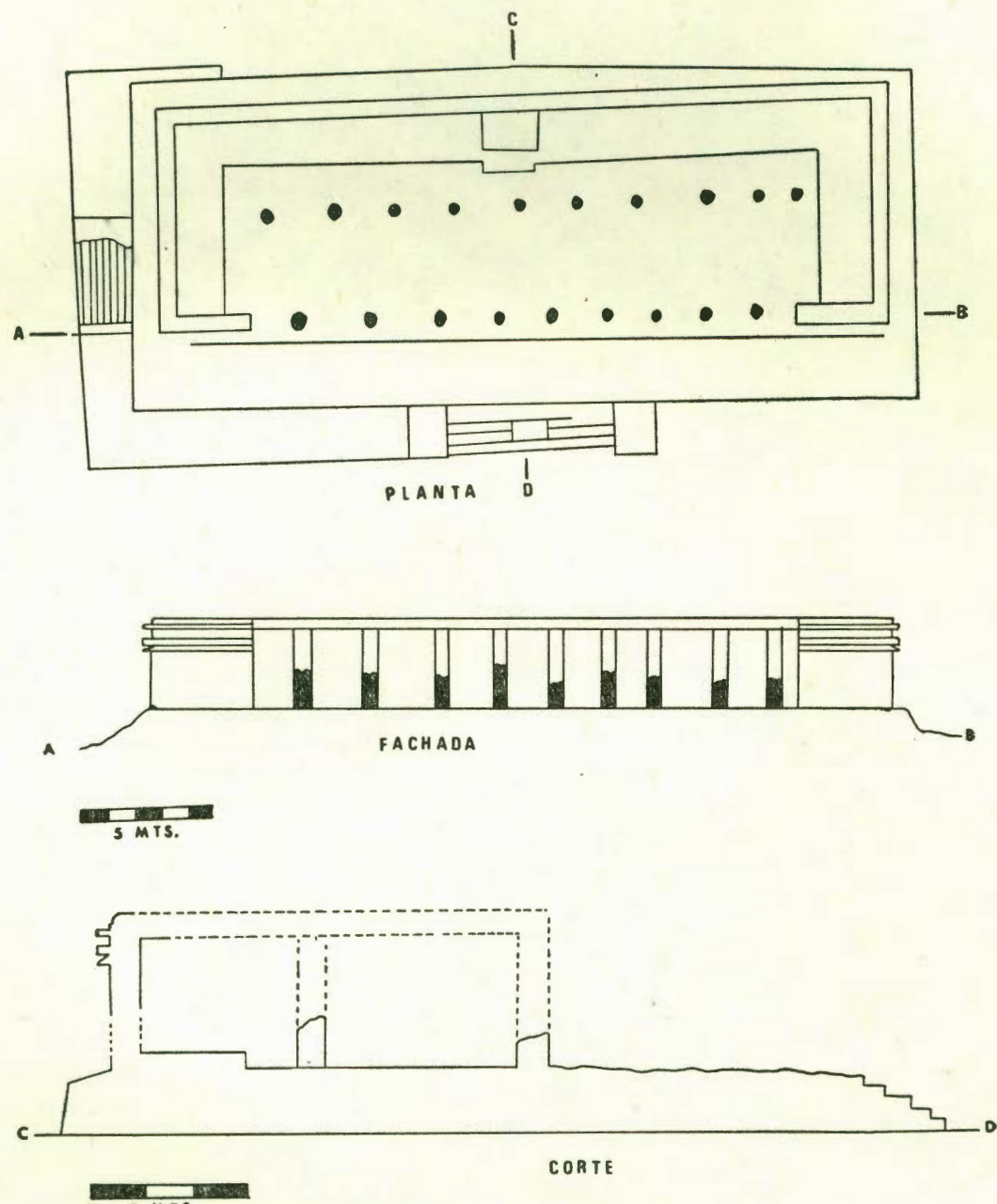

3 MTS. ESTRUCTURA

F IG. 4

existe un altar en su parte media, mientras que en la estructura 7 tenía un personaje en el centro de la escalinata. En la parte norte de la península de Yucatán son varios los ejemplos de estructuras en forma de "L"; en Can Cun la estructura 1 de El Rey (figura 5) y otra de San Miguel, en Tulum la estructura 21, en Cozumel la estructura 5a. del Grupo I de San Gervasio y en Mayapán la estructura 54. Por el contrario estructuras de doble columnata, escalinata al centro, con banqueta y altar interior son muy características del Rey, San Miguel y Mayapán, baste recordar las estructuras $66,145,151,164,212$ etcétera de este último. 

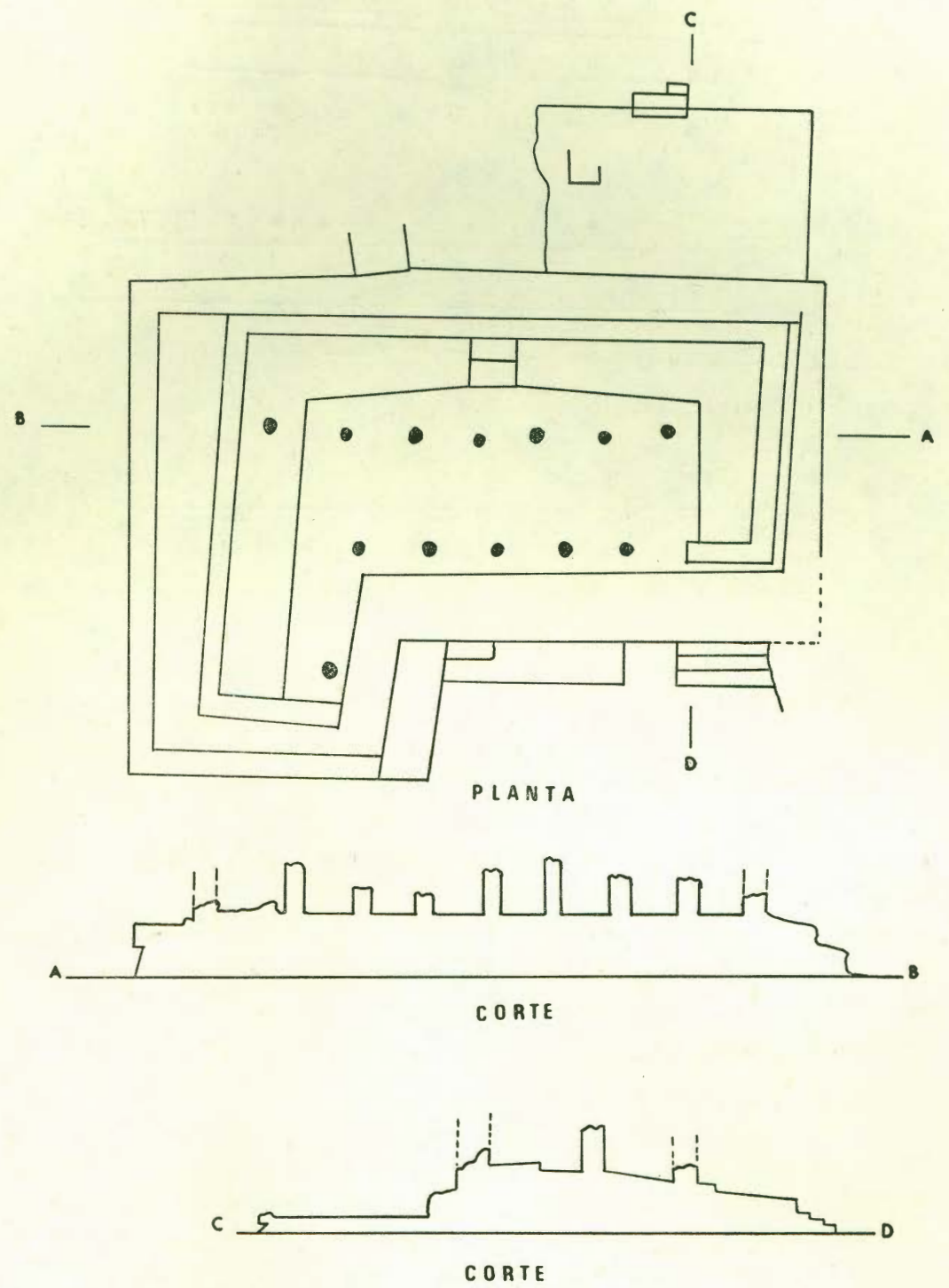

ESTRUCTURA I

FIG. 5 
En el lado norte perteneciente al conjunto El Rey, se localizan 9 plataformas que tienen una altura promedio de $0.60 \mathrm{cms}$. y llegan a medir en promedio 12 metros de largo por 8 de ancho. Todas estas plataformas tienen una escalera de acceso con alfardas, el cuerpo de dichas plataformas se compone de talud y cornisa o muro vertical.

Estas plataformas se distribuyen de tal manera que forman una calle o calzada interrumpida por pequeños altares, que están al frente de las plataformas.

Pinturas. Este conjunto se localiza al sur de El Rey y su nombre se debe a la existencia de pinturas murales; en la actualidad no existen, sin embargo, durante las exploraciones se encontraron restos que confirmaron su existencia.

El conjunto se compone de 27 estructuras divididas en plataformas, edificios ceremoniales y/o civiles o palacios y altares. La distribución es parecida al lado norte, pues las estructuras se alinean a ambos lados de una calle o calzada, en la cual también existen altares frente a los edificios más importantes.

Dignas de una descripción detallada son los llamados palacios, características de la costa oriental; todas estas estructuras están compuestas de dos cuartos comunicados entre sí y con un acceso pequeño en la parte posterior. Al frente tienen una escalinata con alfardas y los muros que forman la plataforma tiene talud y corniza. A la entrada del primer cuarto existen dos columnas o pilastras, más parte de la pared frontal, adornada con detalles característicos de la región; en su interior pueden existir o no columnas y una banqueta con un altar central. El segundo cuarto es de menores dimensiones y se comunica con el primero por una puerta que está cerca del altar. Todas estas estructuras conservan restos del techo plano y están adornados con molduras en su exterior. (Plano 1 , conjunto pinturas).

Este tipo de estructuras con algunas variaciones existen también en Tulum, San Miguel y San Gervacio en Cozumel. En Tulum las estructuras 34 y 35 no tienen columnas en su interior y el altar o adoratorio está siempre en el segundo cuarto, la banqueta aparece tanto en el primero como segundo cuarto, mientras que en Can Cun nunca aparece en el segundo cuarto. Las otras características son muy parecidas. Hay que hacer notar que este tipo de edificio no se conoce ni en Chichén Itzá ni en Mayapán.

Todas estas plataformas y "palacios" fueron construidos utilizando un rasgo que acusa marcada influencia mexicana y que es 

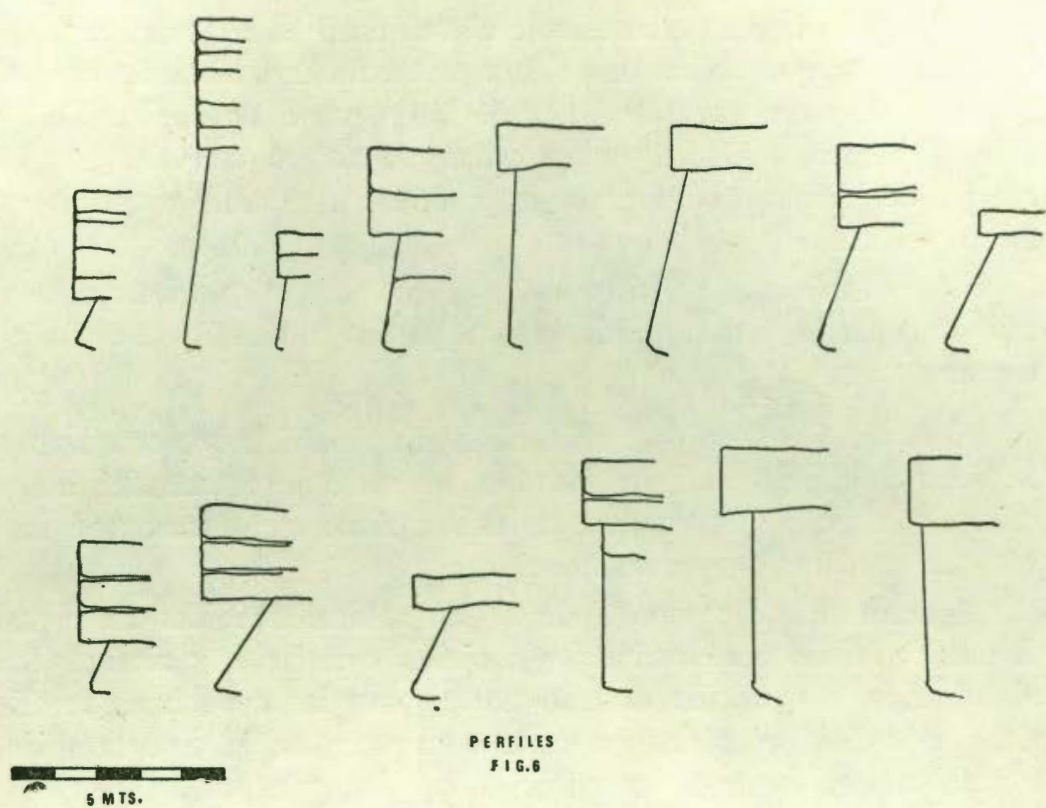

muy característico de ambos sitios, el talud y cornisa o muro recto y cornisa (figura 6), lo mismo que la escalera con alfardas rematadas en dado.

El Rey presenta en su parte central un pequeño centro ceremonial con dos plazas al que se llega por dos calles, la más importante al sur y otra secundaria al norte.

San Miguel. Al norte, a un kilómetro aproximadamente de EI Rey, se localiza San Miguel con 30 estructuras muy claras, además de otros restos arquitectónicos. Su distribución es lineal y cubre una extensión de 600 metros de norte a sur por 80 metros de ancho (plano II).

Tentativamente para su descripción, dividiré el sitio en tres conjuntos: conjunto de la gran pirámide, conjunto central o de los dragones y conjunto norte.

La arquitectura de San Miguel es típica de la tradición arquitectónica denominada costa oriental. Aunque quedan pocos restos, se pueden apreciar las molduras de los edificios, parecidos a los descritos para El Rey, Tulum, Xcaret etcétera.

Conjunto de la Gran Pirámide. Consta de una estructura piramidal, un palacio muy parecido a los de Pintura y seis plataformas, una de ellas con un chultún en el centro. 
Solamente en este conjunto se han realizado trabajos de exploración y consolidación. La estructura piramidal de altura mide 8 metros, 26.50 metros de frente por 23.50 metros de largo, es la estructura más alta de la isla. Durante las exploraciones se determinaron tres etapas de construcción.

Conjunto central o de los dragones. El nombre de los dragones se debe a la existencia de una estructura con dos cabezas de serpientes bastante destruidas, al pie de las alfardas de una escalera.

Este conjunto se compone de cinco edificios con columnas rodeando una gran plaza; en ella existen pequeñas plataformas y cuartos; en una de las estructuras se encontró casualmente una escultura de una serpiente emplumada hecha en basalto, existe además restos de otra cabeza de serpiente.

Conjunto norte. Se compone básicamente en una gran plataforma de doble columnata, con alfardas y escaleras al frente; tres estructuras con columnas en el interior y seguramente techo plano; 9 plataformas y algunos altares.

Sobre la duna, mirando hacia el mar, existe una estructura muy destruida por el saqueo, que acaso pudo haber servido como punto de vigilancia.

En San Miguel existen una serie de elementos que sugieren la posibilidad de una ocupación anterior al año 1200 D.C. sin embargo, por la información obtenida a través de la arquitectura, cerámica y pintura mural de Can Cun, se hace evidente que la mayor ocupación corresponde al postclásico tardío (1200-1520 D.C.)

Los sitios El Rey y San Miguel participan en la tradición denominada costa oriental, caracterizada por los siguientes rasgos: pequeños templos de una sola crujía, o bien templos sobre basamentos piramidales de varios cuerpos, uso del dintel remetido, burdo labrado de piedra, recubiertos por un aplanado de estuco, techos planos con vigas y mortero, frecuente uso de columnas, talud y cornisa o muro recto y cornisa, molduras en la parte superior de los edificios, escalinatas con alfardas rematadas con dados, pintura mural, basamentos circulares, columnas serpentinas, chac mol, greca escalonada y serpientes emplumadas. Estos serían, entre otros menos comunes los rasgos arquitectónicos más sobresalientes que, como han dicho Spinden (1957), Lothrop (1924), Ruz L. (1964), acusan una marcada influencia mexicana trasmitida a través de la cultura tolteca de Chichén Itzá y más directamente asociada a Mayapán. 
Por otra parte, otros autores creen que los rasgos de la costa oriental son anteriores en la cultura maya y Piña Chán (1972) cree que en Yucatán hay un desarrollo del estilo Puuc, cuyo origen está en los Chenes, y este a su vez, en el de río Bec; tradiciones o estilos arquitectónicos ocurridos durante el clásico, pero que los conceptos arquitectónicos de la columna, atlantes, serpientes emplumadas, símbolos de venus etcétera, ya existían en la cultura maya, y que junto con otros llegados de la costa del golfo y Guatemala crean al estilo Chichén, que posteriormente será el estilo arquitectónico de la península.

Sea cual fuere la causa de este cambio en los conceptos religiosos y arquitectónicos de la península de Yucatán, y específicamente en Chichén Itzá, lo importante es que se dieron una serie de elementos que caracterizan esa cultura durante el postclásico temprano y que, a partir del 1200 D.C. hay una consolidación de elementos que se incorporaron definitivamente a la cultura maya de la península de Yucatán desechando otros, tal como los tenemos presentes en Mayapán y en la costa oriental de Quintana Roo.

Comentarios. Excepto por El Conchero, excavado por Andrews IV, y Koxolnah, sitio ubicado muy cerca de la isla, no se tienen evidencias ocupacionales más tempranas. Durante el periodo clásico no existen, o no se han encontrado evidencias arqueológicas en la isla; los únicos reportados para este periodo en la costa oriental podrían ser El Meco, Xelha y Akumal.

La costa oriental de Quintana Roo durante el postclásico temprano sigue siendo todavía un área de poca importancia si se le compara con los sitios del postclásico tardío (Sabloff y Rathje, 1975a; Andrews IV et Andrews T., 1975), ya que las evidencias arquiteçtónicas, el estilo de las pinturas murales y la cerámica, muestras que la mayor ocupación corresponde precisamente al postclásico tardío (1200-1520 D.C.).

Dar una explicación al auge de la costa oriental y al hecho de que estas poblaciones hayan preferido establecerse en áreas costeras, en vez de áreas favorables para la agricultura, puede deberse muy bien a diferentes factores; de ahí que sea necesario plantearse varias alternativas para la explicación del desarrollo de esta área: (Langet 1971).

a) la agricultura basada en el sistema de roza no podía soportar una población demasiado alta durante el postclásico y por tanto el resto de la población se vio obligado a buscar otros medios de subsistencia. 
b) que ya desde antes, los habitantes de la península de Yucatán basaban su subsistencia en la explotación de los recursos marinos, ricos en proteínas, completando su dieta con maíz, tubérculos y cacería.

c) o bien, el auge de esta región pudo deberse a la actividad marítima desarrollada por los mayas en íntima relación con el control del comercio.

Estas podrían ser algunas alternativas viables que nos permitirían comprender mejor el por qué de su auge, ya que durante el postclásico temprano, esta área, al parecer, carecía de importancia. Los sitios reportados son pocos y pequeños; en cambio, a partir de la caída de Chichén Itzá, la costa oriental adquirió importancia y durante el auge de Mayapán se consolida y llega a su apogeo el estilo arquitectónico denominado costa oriental.

Este desarrollo no pudo basarse tan solo en la agricultura, ya que ésta no aseguraba una base económica estable para su existencia y crecimiento, y por lo tanto su apogeo sobrepasó los límites de la base agrícola, como se puede inferir de las siguientes apreciaciones:

a) estas costas e islas no podían haber sostenido un gran número de pobladores a base de agricultura; casi todas ellas están formadas por fajas angostas de arena y depósitos rocosos impropias para la siembra. Por supuesto que esto no es una limitación ya que ninguna isla se encuentra tan lejos de la costa como para que imposibilitara el tránsito diario con tierra firme, sin embargo, tampoco las costas son muy aptas para la siembra.

b) aún en áreas favorables, la siembra del maíz tropieza con serios problemas, la sequía, plagas, el deterioro o pérdida de granos almacenados, son problemas imprevistos que afectan grandemente a la población, y por lo tanto creo que el punto de vista tradicional, de que el maíz era la base de la subsistencia, parece no ser el adecuado para explicar el desarrollo de esta parte de la región maya, aunque sí un factor importante en su dieta.

c) por consiguiente, considero que los datos encontrados durante los exploraciones de El Rey, me permiten inferir, a pesar de la inexistencia de un estudio específico sobre los restos óseos de animales, que la base de la alimentación de esas gentes era la pesca, completamente con la agricultura y la cacería. Se hallaron gran cantidad de huesos de tortuga, dientes de tiburón, esqueletos y huesos aislados de diferentes especies de peces, caracoles y conchas; pesas hechas de tiestos para redes; astas de venado y dientes de jabalí entre otros todavía no identificados.

La península de Yucatán y específicamente la región que aquí me ocupa, tuvo y tiene un claro acceso a los recursos marinos; el establecimiento costero ofrece las ventajas combinadas de un extenso 
número de nichos ecológicos, accesibilidad y facilidad de tránsito, que permitieron su desarrollo. Cardós (1959) indica la existencia de un extenso tránsito de canoas mayas a lo largo de la costa del Caribe, desde la parte norte de Yucatán hasta Honduras; y en el Golfo de México a lo largo del litoral oeste de la península de Yucatán hasta Tabasco. Thompson (1964) señala que después de la Conquista se detuvo el tráfico de plumas y de oro, pero el comercio de la sal continuó, y la sal para la cuenca del Usumacinta y Belice se obtenía del norte de Yucatán, lo cual refleja la importancia del comercio entre los mayas de la península.

Por las evidencias arqueológicas podemos concluir que los habitantes de Can Cun y en general los de la costa, aprovecharon su acceso a los recursos marítimos para su explotación. La tortuga es abundante en la región, pues además de ser un buen alimento, los carapachos tienen diversos usos. El tiburón fue también codiciado no sólo por su carne, sino también por sus dientes que co. múnmente se utilizaban como ofrendas votivas. Las conchas y caracoles también desempeñaban una función en la sociedad maya, como alimento y como materia prima para hacer diversos instrumentos; el copal, las mantas tejidas, la cera, la miel, la grana etcétera, eran productos de Yucatán que se explotaban para ser cam. biados por algunas otras materias y productos de los cuales carecían; jade, cobre, obsidiana, oro, etcétera (Cardós, 1959: 128) de los cuales hay evidencias arqueológicas en la isla de Can Cun.

No existen dudas de que los mayas poseían la habilidad técnica necesaria para explotar los recursos marinos y que la aprovecharon para establecer un intercambio regular con otras regiones. Por lo tanto las principales ocupaciones a lo largo de la costa fueron la pesca, la recolección de la sal y el comercio. Antes y después del año de 1600 D.C. la pesca y el comercio continuaban siendo factores importantes en la economía local y contribuyeron a su desarrollo.

\section{BIBLIOGRAFIA}

ANDREWS, E. WILLYS, IV.

1974 Excavation of an early shell midden on Isla Cancun, Quintana Roo, Mexico. Tulane University, Middle American Research Institute, Pub. 31, pp. 147-97. 
Andrews E. Willys IV and Antony P. Andrews.

1975 A preliminary Study of the ruins of Xcaret, Quintana Roo, Mexico. Tulane University, Middle American Research Institute, Pub. 40.

Arnold, Channing and F.J.T. Frost.

1909 The American Egypt: A record of travel in Yucatan. London.

Atlas Arqueológico de la República Mexicana.

1939 Instituto Nacional de Antropología e Historia. Instituto Panamericano de Geografía e Historia, Pub. 41, México.

Cardós de Méndez, Amalia.

1959 El Comercio entre los Mayas Antiguos. Acta Antropológica, ep. 2, tomo II, No. 1, México.

Chapman, Anne.

1959 Puertos de intercambio en Mesoamérica prehistórica. Instituto Nacional de Antropología e Historia, Serie Historia, No. 3, México.

Fernández, Miguel Ángel.

1945 "Las Ruinas de Tulum. II" Anales del I.N.A.H. I: 95-105. México.

Lange, Frederich W.

1971 Marine Resources: a viable subsistance alternative for the prehistoric lowland Maya. American Anthropologist, 73, pp. 619-39.

Lothrop, SAMUel K.

1924 Tulum: An archaeological study of the east coast of Yu. catan. Carnegie Institution of Washington, Pub. 335.

Piña Chan, Román.

1972 Historia, Arqueología y Arte Prehispánico. Fondo de Cultura Económica, México.

PolLOck, H.E.D.

1962 Mayapan, Yucatan, Mexico. Carnegie Institution of Wash. ington, Pub. 919.

Ruz Lhuillier, Alberto.

1964 Influencias mexicanas sobre los Mayas. En: Desarrollo Cultural de los Mayas, E.Z. Vogt y A. Ruz Lhuillier, eds. Centro de Estudios Mayas, U.N.A.M. pp. 195-227, México.

Sabloff, Jeremy A. and W.L. Rathje.

1975a Changing Pre-Columbian Commercial Systems. Harvard University Cambridge, Massachussetts, No. 3.

1975b The Rise of Maya Merchant Class. Scientific American, Vol. 73, pp. 619-39.

SANDERs. William T.

1955 An archaeological reconnaissance of northern Quintana Roo. Carnegie Institution of Washington, Current Reports, 2 (24): 179-219.

1962 Cultural ecology of the Maya lowlands. Part I Estudios de Cultura Maya 2: 79-121. 
1963 Cultural ecology of the Maya lowlands. Part II Estudios de Cultura Maya, 3: 203-41.

SPINDEN, Herbert.

1957 Maya Art and Civilization. Indian Hills, Colorado.

STePHens, John L.

1843 Incidents of travel in Yucatan. 2 vols. New York.

Thompson, J. ERIC S.

1964 Trade relations between the Maya highlands and lowlands.

Estudios de Cultura Maya, 4: 13-49. 


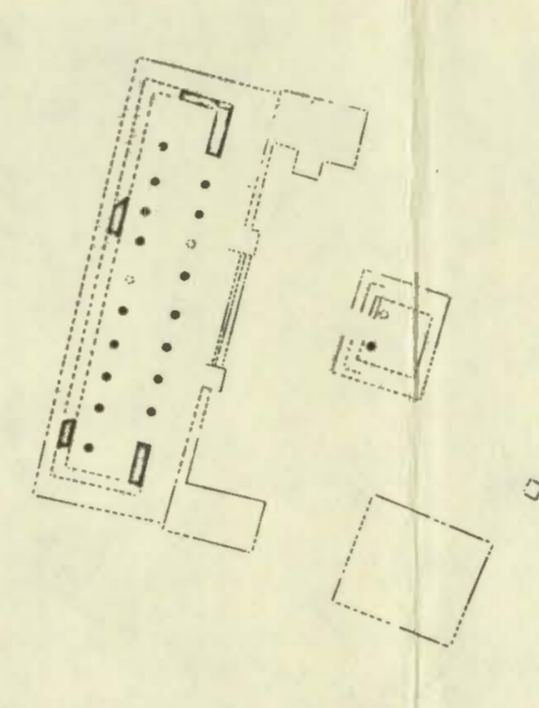

CONJUNTO NORTE

INAH

CENTRO REgIONAL DEL SURESTE PROYECTO: CAN CUN-SAN MIGUE TOPOGRAFIAY DIBUJO ERNESTO VARO
1970

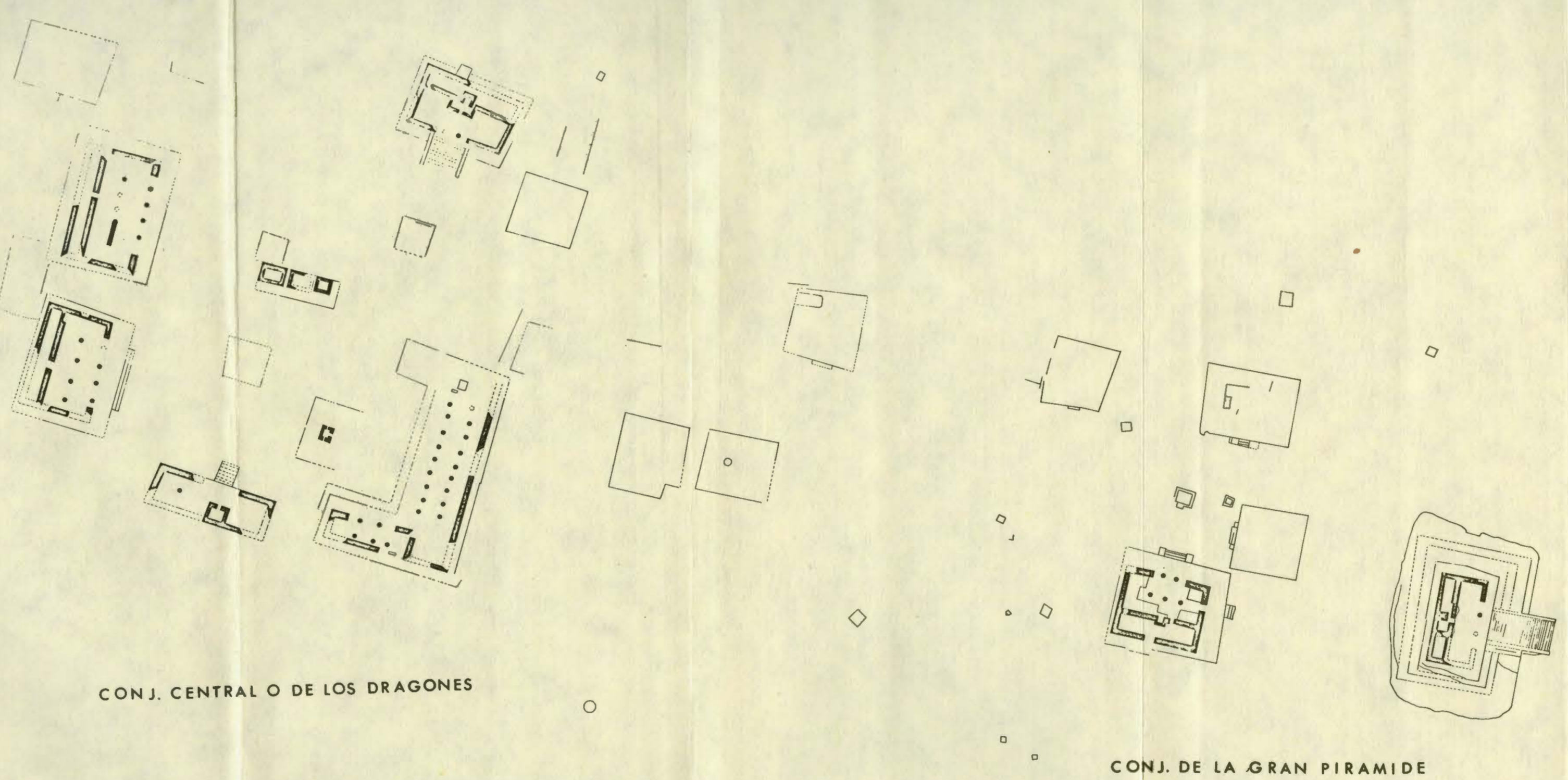




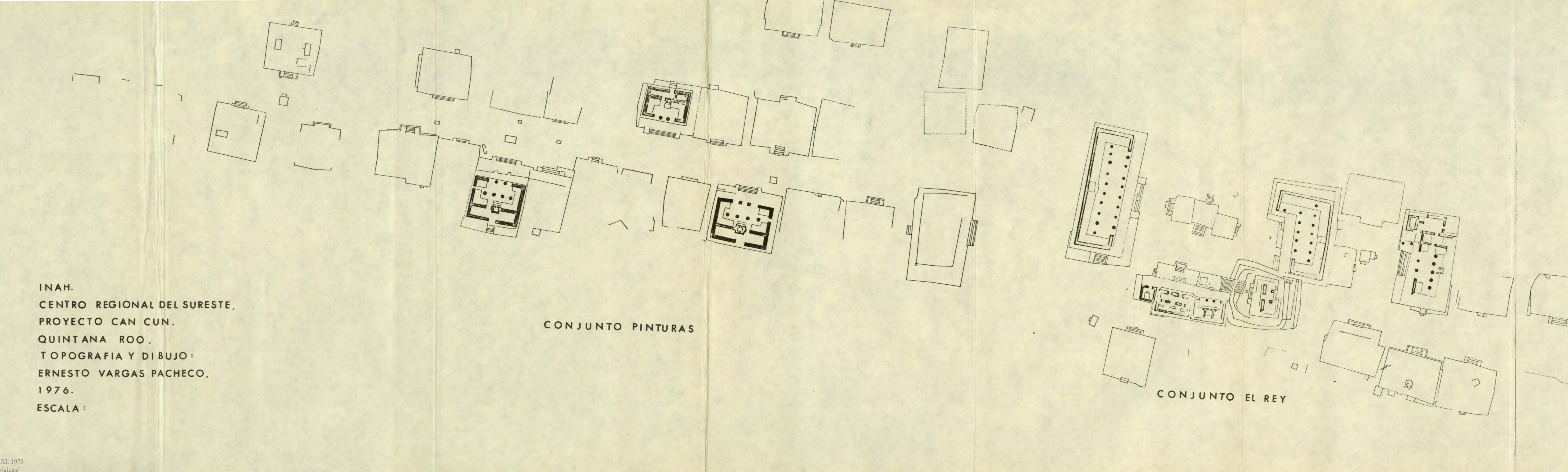

